unterschiedlicher Weise geschützt sind. ${ }^{62}$ Ein Zusammenbruch der IWC scheint unwahrscheinlich, zumal der Austritt eines ausgesprochenen Wal-Nutzungsstaates andere Staaten motivieren könnte, der IWC nun, im Interesse des Walschutzes, beizutreten. Die verbleibenden Staaten werden in aller Wahrscheinlichkeit nach den Kompass der IWC weiter im Sinne des Walschutzes ausrichten.

Open Access. Dieser Artikel wird unter der Creative Commons Namensnennung 4.0 International Lizenz veröffentlicht, welche die Nutzung, Vervielfältigung, Bearbeitung, Verbreitung und Wiedergabe in jeglichem Medium und Format erlaubt, sofern Sie den/die ursprünglichen Autor(en) und die Quelle ordnungsgemäß nennen, einen Link zur Creative Commons Lizenz beifügen und angeben, ob Änderungen vorgenommen wurden.
Die in diesem Artikel enthaltenen Bilder und sonstiges Drittmaterial unterliegen ebenfalls der genannten Creative Commons Lizenz, sofern sich aus der Abbildungslegende nichts anderes ergibt. Sofern das betreffende Material nicht unter der genannten Creative Commons Lizenz steht und die betreffende Handlung nicht nach gesetzlichen Vorschriften erlaubt ist, ist für die oben aufgeführten Weiterverwendungen des Materials die Einwilligung des jeweiligen Rechteinhabers einzuholen.

Weitere Details zur Lizenz entnehmen Sie bitte der Lizenzinformation auf http://creativecommons.org/licenses/by/4.0/deed.de.

Open Access funding provided by University of Helsinki including Helsinki Universtiy Central Hospital.

62) Jefferies, Marine Mammal Conservation and the Law of the Sea, 2016

\title{
https://doi.org/10.1007/s10357-020-3760-8 \\ Braucht Deutschland ein Kunststoffgesetz? Eine Analyse am Beispiel der Kunststoffverpackungen
}

\section{Björn Schwan*}

(c) Der/die Autor(en) 2020. Dieser Artikel ist eine Open-Access-Publikation.

Die Behandlung von Kunststoffabfällen wird immer wieder diskutiert. Auch die jetzige Novelle des Kreislaufwirtschaftsgesetzes (KrWG) betrifft diesen Bereich. Bedarf es eines eigenen Kunststoffgesetzes? Diese Frage wird auf der Basis der bisherigen Regelungen untersucht.

\section{Einführung}

Kunststoffe, synonym auch Plastik genannt, sind allgegenwärtig und gelten zu Recht als Inbegriff der Wegwerfgesellschaft. Sie zeichnen sich durch viele positive Eigenschaften aus, denn sie sind leicht, billig, nahezu beliebig formbar und können für den jeweiligen Einsatzzweck maßgeschneidert werden, was sie zum idealen Material u.a. für Verpackungen und Einwegprodukte macht. Aber es gibt auch die problematische Kehrseite, die sich mit drei Stichworten umreißen lässt: Menge, Umwelt und Gesundheit.

Von 1950 bis 2015 wurden weltweit rund 8,3 Milliarden Tonnen Plastik produziert. Nur rund $7 \%$ dieser gewaltigen Menge wurde recycelt, wobei nur aus rund 100 Millionen Tonnen (ca. 1,2\%) tatsächlich neue Produkte aus recyceltem Kunststoff hergestellt wurden. Der weitaus größte Anteil der Kunststoffproduktion entfällt auf Verpackungen und Einwegprodukte und bei der Entsorgung überwiegt weltweit mit rund $79 \%$ der Anteil an Kunststoffen, welcher nach der Nutzung deponiert und nicht weiter behandelt wurde bzw. achtlos in der Natur gelandet ist. Wissenschaftliche Szenarien gehen davon aus, dass bis zum Jahr 2050 kumuliert ca. 25 Milliarden Tonnen an Plastikabfällen angefallen sein könnten. ${ }^{1}$ Sollten sich diese Prognosen bewahrheiten, würden dann 20 Prozent des Erdölkonsums und 15 Prozent der Treibhausgase auf die Kunststoffproduktion entfallen. ${ }^{2}$ Und auch Deutschland ist ein wichtiger Produzent, der 2018 mit einer Jahresproduktion von

Björn Schwan, M.Sc. RWTH,

Umweltoberinspektoranwärter beim Land Nordrhein-Westfalen, Aachen, Deutschland
19,3 Millionen. Tonnen mit rund 5,4\% an der weltweiten Produktion beteiligt war. ${ }^{3}$

Diese gewaltigen Mengen verursachen weltweit erhebliche Umweltprobleme. So könnten mittlerweile bereits 86 Millionen. Tonnen Kunststoffabfälle in den Weltmeeren gelandet sein. ${ }^{4}$ Da Kunststoffe durch Abrieb und vor allem durch nicht sachgerechte Entsorgung im Laufe der Zeit zu Mikroplastik zerfallen, wurden mittlerweile Plastikpartikel überall dort gefunden, wo auch danach gesucht wurde, selbst an arktischen Stränden, in der Tiefsee oder auf den höchsten Alpengipfeln. ${ }^{5}$ Mikroplastik kontaminiert Wasser, Böden und Luft und ist bereits umfassend in der Nahrungskette angekommen. ${ }^{6}$

Plastik gefährdet mittlerweile die menschliche Gesundheit umfassend und auf globaler Ebene. Es ist davon auszugehen, dass bis zu 5 Gramm Mikroplastik pro Woche über die Aufnahme von Wasser, Nahrung und Atemluft in den menschlichen Körper gelangen können. ${ }^{7}$ Auch wenn es umfangreiche gesetzliche Regelungen für Verpackungen mit Lebensmittelkontakt gibt, so steht dennoch fest, dass sehr geringe Mengen der Inhaltsstoffe von Plastikverpackungen in aller Regel auch auf das verpackte Lebensmittel übergehen, da Kunststoffe nicht inert sind. Aktuell hat die Forschungs-

^) Der Aufsatz basiert auf der im März 2020 verfassten, deutlich umfangreicheren Masterarbeit gleichen Titels im Fach Umweltingenieurwissenschaften an der RWTH Aachen.

1) Geyer/Lambeck/Law, Production, use, and fate of all plastics ever made, Science Advances 3 (2017), e1700782, S. 1 ff.

2) Röchling Stiftung GmbH (Hrsg.), Polyproblem Kunststoff und Umwelt, S. 9

3) PlasticsEurope, Plastics - the Facts 2018, S. 19

4) Heinrich-Böll-Stiftung (Hrsg.), Plastikatlas, S. 29

5) Bergmann, White and wonderful? Microplastics prevail in snow from the Alps to the Arctic, Science Advances 5/2019.

6) Cox et al., Human Consumption of Microplastics, Environmental Science \& Technology 12/2019, S. $7068 \mathrm{ff}$.

7) WWF (Hrsg.), Aufnahme von Mikroplastik aus der Umwelt beim Menschen. 
gruppe „PlastX“" 34 Alltagskunststoffe genauer untersucht, deren Inhalte in irgendeiner Form mit dem menschlichen Körper in Berührung kommen, z.B. Softdrink-Flaschen, Kaffeebecher, Aufbewahrungsbehälter, aber auch Duschgel und Taschentücherverpackungen. ${ }^{9}$ Insgesamt wurden 1411 Inhaltsstoffe bei der Analyse entdeckt, in einzelnen Produkten sogar mehrere Hundert. Dabei wurden über $80 \%$ der gefundenen Stoffe, also mehr als 1100 davon, als nicht identifizierbar beschrieben, da sie als Abbau- und Nebenprodukte erst im Herstellungsprozess entstehen. Bedenklich ist, dass es nicht bekannt ist, welche dauerhaften Auswirkungen die einzelnen Inhaltsstoffe im Körper verursachen und welche gesundheitsschädlichen Folgen sie in ihrer Kombination bzw. Wechselwirkung haben. Bestimmte chemische $\mathrm{Zu}-$ satzstoffe wie z.B. Bisphenol A oder bestimmte Phthalate (Weichmacher) gelten als hormonaktiv und stehen in Verdacht, bereits in sehr geringen Konzentrationen, die weit unter den geltenden Grenzwerten für eine Toxizität liegen, erhebliche Auswirkungen auf das Fortpflanzungs-, Nerven-, Immun- und Herz-Kreislauf-System zu haben; weiterhin könnten sogar Krankheiten wie Adipositas, Diabetes oder Krebs ausgelöst werden. ${ }^{10}$

Viele Lösungsalternativen liegen auf der Hand, wie die Vermeidung oder Substitution von Plastikprodukten, die Wiederverwendung, das konsequente Recycling und der Umstieg in eine echte Kreislaufwirtschaft. Nachfolgend soll untersucht werden, ob und inwieweit der in der Bundesrepublik Deutschland geltende Rechtsrahmen im Hinblick auf Kunststoffverpackungen und Kunststoff-Einwegprodukte geeignet ist, die richtigen, lösungsorientierten Impulse zu setzen und welche weitergehenden Handlungsbedarfe bestehen.

\section{Strategien gegen Kunststoffverpackungen}

Aktuell wird weltweit im politischen Bereich über das Plastikproblem nachgedacht und es gibt eine Reihe von wichtigen Strategieaussagen und Initiativen, die auch für die deutsche Politik und Gesetzgebung im Umweltbereich von Bedeutung sind.

\subsection{Politischer Rahmen der United Nations (UN)}

Im Rahmen der UN gibt es zahlreiche Initiativen zur weltweiten Förderung der Nachhaltigkeit.

Die Agenda 2030 für Nachhaltige Entwicklung wurde am 25. 9. 2015 beim UN- Nachhaltigkeitsgipfel der Staatsund Regierungschefs verabschiedet. Diese stellt eine globale Richtschnur für das Handeln der Nationen dar und gilt als Meilenstein der internationalen Zusammenarbeit. Die Frage nach der Rechtsnatur der Agenda 2030, völkerrechtlicher Vertrag oder nur ,soft law“"11, kann hier dahinstehen, da sich die Europäische Union (EU) und auch Deutschland zu einer umfassenden Transformation verpflichtet haben. Eine der fünf Kernbotschaften der Agenda 2030 lautet: „Wir sind entschlossen, den Planeten vor Schädigung $\mathrm{zu}$ schützen, unter anderem durch nachhaltigen Konsum und nachhaltige Produktion, die nachhaltige Bewirtschaftung seiner natürlichen Ressourcen und umgehende Maßnahmen gegen den Klimawandel, damit die Erde die Bedürfnisse der heutigen und der kommenden Generationen decken kann." ${ }^{12}$ Alle in dieser Aussage genannten Aspekte sind gleichzeitig auch zu beachtende Indikationen für den Umgang mit dem Thema Plastik.

Bereits 2016 hat die Bundesregierung einen Bericht zum High-Level Political Forum on Sustainable Development 2016 herausgegeben ${ }^{13}$, in dem sie sich zu einer ,ambitionierten Umsetzung der Agenda" bekennt. Die Agenda 2030 müsse in allen Lebensbereichen umfassend transformiert werden. Besonders relevant ist Ziel 12 der Agenda 2030 „Nachhaltige/r Konsum und Produktion“. In diesem Kontext verpflichtet sich die Bundesregierung u.a. zur
Steigerung der Ressourceneffizienz entlang der gesamten Wertschöpfungskette“ sowie zur „Umsetzung des Abfallvermeidungsprogramms des Bundes und zur Weiterentwicklung der Kreislaufwirtschaft".

Die Umweltbehörde der UN, das UN Environment Programme (UNEP), veröffentlichte 2018 die Studie „SINGLE-USE PLAȘTICS - A Roadmap for Sustainability“. ${ }^{14}$ Darin werden Überlegungen zur Eindämmung der Einweg-Plastik-Flut dargelegt und positive Beispiele aus verschiedensten Weltregionen vorgestellt. Das Dokument enthält die Feststellung, dass die weltweiten Mengen an Kunststoffabfällen nicht mehr zu bewältigen seien, es sei denn, es würde die Art und Weise überdacht und geändert, wie Kunststoffe hergestellt, verwendet und gemanagt werden. Um eine der größten Umweltbelastungen unserer Zeit $\mathrm{zu}$ bewältigen, sei es erforderlich, dass Regierungen regulierend eingreifen, Unternehmen innovativer werden und Einzelpersonen handeln.

Es gibt noch weitere aktuelle Initiativen unter der Ägide des UNEP, so z.B. „The New Plastics Economy Global Commitment". ${ }^{15} \mathrm{Zu}$ den Forderungen dieser Initiative gehören im Verpackungsbereich die Abschaffung problematischer und unnötiger Verpackungen sowie die sukzessive Umstellung auf wiederverwendbare, recycelbare und kompostierbare Verpackungen. ${ }^{16}$ Weiterhin wurde eine Vision für eine neue Plastik-Kreislaufwirtschaft vorgestellt, die auch als Vorlage für regierungsseitige Initiativen und Regulierungen dienen kann.

Die vorgestellten weltweiten Initiativen unter dem Dach der UN machen deutlich, dass weltweit ein wachsendes Problembewusstsein entstanden ist und dass mit Nachdruck versucht wird, Lösungen für die Umweltproblematik der Plastikabfälle zu schaffen.

\subsection{Aktuelle politische Initiativen im Rahmen der OECD und der G7}

Die Bundesrepublik Deutschland ist auch Mitglied in verschiedenen internationalen Organisationen und informellen Zusammenschlüssen. Auch dort gibt es in neuerer Zeit Initiativen, Absprachen und Vereinbarungen, die ebenfalls für den politischen Rahmen in Deutschland wesentlich sind.

Die Organisation für wirtschaftliche Zusammenarbeit und Entwicklung (OECD) hat im Mai 2018 ein „Global Forum on Environment - Plastics in a Circular Economy: Design of Sustainable Plastics from a Chemicals Perspective" veranstaltet und sich in diesem Rahmen mit den chemischen Aspekten des Produktdesigns auseinandergesetzt. Wesentliche Ergebnisse waren u. a., dass die chemische $\mathrm{Zu}-$ sammensetzung der Kunststoffprodukte eine entscheidende Rolle im Hinblick auf die Recyclingfähigkeit spiele und dass die Rahmenbedingungen und Märkte für die Re-

8) PlastX, Researching Plastics, Stand 2.2.2020, abrufbar unter http://www.plastx.org/.

9) Zimmermann et al., Benchmarking the in Vitro Toxicity and Chemical Composition of Plastic Consumer Products, Environmental Science \& Technology 2019, 53, S. $11467 \mathrm{ff}$.

10) Bundesamt für Gesundheit (Schweiz), Factsheet Bisphenol A (Stand 5/2019).

11) Huck/Kurkin, ZaöRV 78 (2018), 375, 391

12) United Nations, A/Res/70/1, Resolution ,Transformation unserer Welt: die Agenda 2030 für nachhaltige Entwicklung“, S. 2

13) Bundesregierung, Bericht der Bundesregierung zum High-Level Political Forum on Sustainable Development 2016.

14) United Nations Environment Programme, Giacovelli, SingleUse Plastics: A Roadmap for Sustainability.

15) Ellen MacArthur Foundation, The New Plastics Economy Global Commitment - 2019 Progress Report.

16) Ellen MacArthur Foundation, The New Plastics Economy Global Commitment - 2019 Progress Report, S. 8. 
cyclingindustrie besonders betrachtet und reguliert werden müssten, damit Rezyklate als Sekundärrohstoffe nicht zwangsläufig den Trends und dem Verdrängungswettbewerb der Primärmärkte ausgesetzt seien. ${ }^{17}$

Die G7, ein informelles Forum der Staats- und Regierungschefs aus sieben wichtigen Industrieländern, hat 2018 beim G7-Gipfel in Kanada eine „Oceans Plastics Charter" erarbeitet, die mittlerweile von 26 Regierungen, darunter die Bundesregierung, und 67 Unternehmen und Organisationen als sog. „Partner“ anerkannt wurde. ${ }^{18}$ Auch wenn es dabei inhaltlich vorrangig um den Meeresschutz geht, so gehen die Inhalte weit darüber hinaus. Die Partner verpflichten sich, Maßnahmen für einen umwelt- und ressourcenschonenden Umgang mit Kunststoffen während des gesamten Produktlebenszyklus zu ergreifen. Darunter findet sich z.B. die Forderung, dass zusammen mit der Industrie bis 2030 das Ziel verfolgt wird, dass Plastikprodukte $\mathrm{zu} 100 \%$ wiederverwendbar, recycelbar oder, soweit dies nicht möglich ist, rückgewinnbar sein sollen. Bis 2030 sollen mindestens $55 \%$ der Kunststoffverpackungen wiedergenutzt oder recycelt werden und bis 2040 lautet das Ziel 100\%. Der Rezyklatanteil von Kunststoffprodukten soll um $50 \%$ gesteigert werden, funktionierende Märkte für Sekundärrohstoffe sollen aufgebaut werden und öffentliche Aufträge sollen nach „Green Procurement“-Gesichtspunkten vergeben werden. Und es sollen massiv Kunststoffinnovationen sowie die Forschung und Entwicklung im Hinblick auf nachhaltige Technologien, Design- und Produktionsmethoden gefördert werden.

\subsection{Aktuelle Entwicklungen in China}

Da China mit ca. 30\% Anteil an der weltweiten Plastikproduktion und 39\% Anteil am weltweiten Plastikmüllaufkommen der weltweit bedeutendste Player ist, sind die dortigen politischen Weichenstellungen auch für die EU und Deutschland von großer Bedeutung. ${ }^{19}$ Mit Wirkung ab Januar 2018 wurde der sog. China waste import ban in Kraft gesetzt, ein Importstopp für eine Vielzahl von minderwertigen Kunststoffabfällen.

Im Januar 2020 haben das chinesische Umweltministerium und die Nationale Entwicklungs- und Reformkommission angekündigt, dass ab Ende 2020 weitreichende Verbote für Einweg-Plastikprodukte eingeführt werden. Es sollen dutzende Anlagen zur ,comprehensive resource utilisation“" (umfassenden Ressourcennutzung) aufgebaut werden, womit sowohl das Recycling als auch die Vorbereitung zur Wiederverwendung gemeint sein dürften. ${ }^{20}$ China bewegt sich also massiv in Richtung Umweltschutz und Nachhaltigkeit und könnte dabei sicher auch eine technologische Führung anstreben.

\subsection{Politischer Rahmen der EU}

Der von der EU gesetzte Rahmen ist von erheblicher Bedeutung für die Mitgliedsländer und ist für Überlegungen zur Weiterentwicklung des deutschen Umwelt- und Abfallrechts umfassend zu beachten.

\subsubsection{Grünbuch zu einer europäischen Strategie}

für Kunststoffabfälle in der Umwelt

Bereits 2013 hat die EU dieses Grünbuch herausgegeben $^{21}$, das sich auch heute noch erstaunlich aktuell liest. Konstatiert wurde u.a., dass Kunststoffabfälle noch nicht ausdrücklich im EU-Abfallrecht geregelt waren, dass Kunststoffabfälle noch nicht als gefährlich eingestuft waren und dass Kunststoff über das größte Potenzial zur Verringerung der Umweltauswirkungen von Abfall verfüge; und auch das Problem der gefährlichen Zusatzstoffe im Kontext von Gesundheit und Recyclingfähigkeit wurde gesehen.

\subsubsection{Den Kreislauf schließen - Ein Aktionsplan} der EU für die Kreislaufwirtschaft

Die Kommissionsmitteilung von 2015 enthält zahlreiche Aussagen zu Kunststoffen und Kunststoffabfällen. ${ }^{22}$ Es wird die Bedeutung der stofflichen Qualität bzw. der Verunreinigungsgrade für ein hochwertiges Recycling angesprochen, wofür EU-weite Standards geschaffen werden müssten. Auch sei eine einheitliche rechtliche Sichtweise wichtig, ab wann ein Sekundärrohstoff nicht länger als „Abfall“" zu betrachten sei. Weiterhin wurde ein - bislang noch nicht erfolgtes - Follow-up zum Grünbuch zu Kunststoffabfällen angekündigt, in das eine neu auszuarbeitende Strategie einfließen soll, ,um die von Kunststoffen ausgehenden Probleme entlang der gesamten Wertschöpfungskette und unter Berücksichtigung ihres gesamten Lebenszyklus zu bewältigen".

\subsubsection{Eine europäische Strategie für Kunststoffe in der Kreislaufwirtschaft}

Anfang 2018 wurde „Eine europäische Strategie für Kunststoffe in der Kreislaufwirtschaft" veröffentlicht. ${ }^{23}$ Darin sieht sich die EU ,,bestens positioniert, um beim Übergang zu Kunststoffen der Zukunft eine Führungsrolle zu übernehmen". ${ }^{24}$

Zentrale Elemente der Kunststoffstrategie sind die Stärkung des Kunststoffrecyclings, mehr Abfälle getrennt sammeln, Kapazitäten zum Recycling in der EU ausbauen und mehr Recyclingkunststoffe verwenden. Chemische Substanzen, die Kunststoffen zugesetzt werden und das Recycling behindern, sollen ersetzt werden, der Export von Kunststoffabfällen soll beendet werden und die Verbraucher sollen durch neue Kennzeichnungen eine bessere Chance bekommen auf Kunststoffe zu verzichten.

\subsubsection{Regelung der Schnittstelle zwischen Chemikalien-,} Produkt- und Abfallrecht

Konkreter wurde die EU 2018 im Rahmen einer Mitteilung zur Umsetzung des Kreislaufwirtschaftspakets „Optionen zur Behandlung der Schnittstelle zwischen Chemikalien-, Produkt- und Abfallgesetzgebung" ${ }^{25}$. Danach müssen Informationen zum Vorhandensein von bedenklichen Stoffen auch für diejenigen, die mit Abfällen umgehen und sie für die Verwertung vorbereiten, jederzeit verfügbar sein. Zudem sollen Abfälle künftig keine Stoffe mehr enthalten, die in neuen Produkten nicht mehr zulässig wären, da diese sich für das Recycling und den Wiedereinsatz nur sehr bedingt eignen. Die EU-Vorschriften zur Abfallentsorgung sind vollständig $\mathrm{zu}$ harmonisieren und es soll EU-weit einheitlich geregelt werden, wann die Abfalleigenschaft endet und wie Abfall zu einem neuen Material und Produkt wird. Zur Steigerung der Bereitschaft zur Verwendung von Sekundärrohstoffen sollen klare Regelungen erarbeitet werden, welche Chemikalien bei der Herstellung und Verwendung und welche Abfälle im Kontext der Abfallbewirtschaftung gefährlich sind. Die klare Aufforderung an die Mitgliedsstaaten lautet, eine Kohärenz zwischen den Chemikalien-, Produkt- und Abfallgesetzen herzustellen.

17) OECD, Governments need to act to encourage plastic recycling markets.

18) G7, Oceans Plastics Charter.

19) Röchling Stiftung GmbH (Hrsg.), Polyproblem Kunststoff und Umwelt, S. 6.

20) The Guardian, China moves to phase out single-use plastics.

21) Europäische Kommission, COM(2013) 123 final.

22) Europäische Kommission, COM(2015) 614 final.

23) Europäische Kommission, $\operatorname{COM}(2018) 28$ final.

24) Europäische Kommission, COM(2018) 28 final, S. 1.

25) Europäische Kommission, COM(2018) 32 final. 


\subsubsection{EU-Richtlinie zur Änderung der Abfallrahmenrichtlinie ${ }^{26}$}

In der im Mai 2018 veröffentlichten Richtlinie (EU) 2018/851 zur Änderung der Richtlinie 2008/98/EG über Abfälle werden konkrete Vorgaben für die Wiederverwendung und das Recycling von Siedlungsabfällen gemacht, zu denen nach der dortigen Definition auch die Kunststoffabfälle gehören; bis 2025 z. B. soll diese Quote bei 55 Gewichtsprozent liegen und bis 2025 soll 65 Gewichtsprozent erreicht werden. Da Siedlungsabfälle auch erhebliche Anteile an Kunststoffen enthalten, müssen in der Folge auch Maßnahmen zur besseren Sortierung von Siedlungsabfällen ergriffen werden.

\subsection{6 Überwachungsrahmen für die Kreislaufwirtschaft}

2018 wurde die Kommissionsmitteilung über einen Überwachungsrahmen für die Kreislaufwirtschaft veröffentlicht. ${ }^{27}$ Dieser umfasst zehn Indikatoren, die sich auf die vier wesentlichen Phasen und Aspekte der Kreislaufwirtschaft verteilen, nämlich Herstellung und Verbrauch, Abfallbewirtschaftung, Sekundärrohstoffe sowie Wettbewerbsfähigkeit und Innovation.

Alle vier Phasen mit den zehn Indikatoren haben auch Bedeutung für die Kunststoffthematik. Eine Erhöhung der Recyclingquoten führt zu einer besseren Selbstversorgung mit Rohstoffen, die öffentliche Auftragsvergabe kann bei bestimmten Produkten im Sinne von „Green Procurement auch bestimmte Rezyklatanteile einfordern. Das Pro-KopfAbfallaufkommen sollte bei stärkerer Müllvermeidung geringer werden und mit guten Verpackungen kann auch die Lebensmittelverschwendung reduziert werden ( $\mathrm{Nr}$. 1-4). Die Recyclingraten sollten insbesondere bei Verpackungsund Kunststoffabfällen möglichst hoch liegen (Nr. 5 und 6). Hohe Recyclingraten bei Kunststoffen führen zu mehr Sekundärrohstoffen (Nr. 7 und 8) und einem entsprechenden Handel. Und nicht zuletzt werden in der Recyclingwirtschaft bei entsprechenden Rahmenbedingungen auch neue Arbeitsplätze entstehen und auch Innovationen mit entsprechenden Patenten hervorgebracht (Nr. 9 und 10).

\subsubsection{EU-Richtlinie über die Verringerung}

der Auswirkungen bestimmter Kunststoffprodukte auf die Umwelt

Im Juni 2019 wurde die Richtlinie (EU) 2019/904 über die Verringerung der Auswirkungen bestimmter Kunststoffprodukte auf die Umwelt veröffentlicht. ${ }^{28}$ Gem. Art. 1 ist das Ziel der Richtlinie, ,,die Auswirkungen bestimmter Kunststoffprodukte auf die Umwelt, insbesondere die Meeresumwelt, und die menschliche Gesundheit zu vermeiden und zu vermindern und den Übergang zu einer Kreislaufwirtschaft mit innovativen und nachhaltigen Geschäftsmodellen, Artikeln und Werkstoffen zu fördern“. Dafür sollen Maßnahmen zu „Verbrauchsverminderung“ (Art. 4) bei Getränkebechern und Lebensmittelverpackungen für Take-away-Gerichte ergriffen sowie „Vermarktungsverbote“ (Art. 5) für bestimmte Einwegkunststoffprodukte (insbesondere Wattestäbchen, Plastikbesteck, Trinkhalme, Rührstäbchen, Luftballonstäbe sowie aus expandiertem Polystyrol bestehende Lebensmittelverpackungen, Getränkebehälter und Getränkebecher) ausgesprochen werden. Die „Produktanforderungen“ (Art. 6) an bestimmte Einwegplastikprodukte werden ausgeweitet, so müssen z. B. ab 2025 PET-Flaschen (Polyethylenterephthalat)zu mindestens 25 Prozent aus recyceltem Kunststoff bestehen. Im Rahmen einer „Erweiterten Herstellerverantwortung“ (Art. 8) sollen die Hersteller u.a. an den Kosten von bestimmten Sensibilisierungsmaßnahmen sowie von Reinigungsaktionen und anschließender Behandlung der Abfälle von bestimmten Einwegkunststoffartikeln beteiligt werden. Und die Verbraucher sollen mit „Sensibilisierungsmaßnahmen“ (Art. 10) besser informiert werden, z.B. im Umgang mit
Einwegkunststoffartikeln oder zu wiederverwendbaren Alternativen für bestimmte Einwegkunststoffartikel.

Die in der Richtlinie aufgeführten Maßnahmen müssen ab bestimmten Stichtagen von den Mitgliedsstaaten umgesetzt werden (Art. 17), so z. B. die Vermarktungsverbote nach Art. 5 ab dem 3. Juli 2021.

\subsection{Politischer Rahmen in Deutschland}

Umweltschutz und Nachhaltigkeit genießen in Deutschland einen hohen gesellschaftlichen, politischen und rechtlichen Stellenwert. Zudem ist Deutschland u.a. als Mitglied der UN, der OECD, der G7 und insbesondere im Rahmen der EU in vielfältiger Weise in die internationalen Umweltschutzaktivitäten eingebunden. Die deutsche Umweltpolitik verfolgt Ziele wie die Beendigung der Wegwerfgesellschaft, die Vermeidung von Einwegplastik, die Unterstützung des europaweiten Verbots von 10 Einweg-Plastikprodukten und die weitere Stärkung des Recyclings. ${ }^{29}$

\subsubsection{Aussagen im Koalitionsvertrag}

Die derzeit regierende große Koalition aus CDU, CSU und SPD hat sich im Koalitionsvertrag u.a. sehr ausführlich zur Nachhaltigkeit, zum Umweltschutz und zur Kreislaufwirtschaft bekannt. ${ }^{30}$ Auch die Umsetzung der Agenda 2030 wird an mehreren Stellen als grundlegende Richtschnur der deutschen Politik erwähnt. ${ }^{31}$ Wichtige Aussagen im Koalitionsvertrag sind, das Prinzip der Nachhaltigkeit umfassend zu beachten und wirksame Maßnahmen zu ergreifen, um „Wasser und Böden besser zu schützen, die Luft sauberer $z u$ halten und unsere Ressourcen im Kreislauf zu führen“.32 "Wir stehen für eine Weiterentwicklung des erfolgreichen deutschen Modells der Kreislaufwirtschaft. Anspruchsvolle Recyclingquoten, Wettbewerb und Produktverantwortung sollen dabei auch künftig die Leitplanken sein. Wir wollen, auch im Rahmen des europäischen Kreislaufwirtschaftspakets und der weiteren Arbeiten an der europäischen Kunststoffstrategie, Abfallvermeidung und Recycling stärken, die Einsatzmöglichkeiten für recycelte Materialien verbessern und entsprechende Anreize sowie mögliche gesetzliche Pflichten prüfen. Daneben wollen wir die Produktverantwortung weiterentwickeln, d.h. Hersteller müssen Langlebigkeit, Reparierbarkeit und Wiederverwendbarkeit stärker berücksichtigen." 33

„Wir werden auch aus Wettbewerbsgründen aufEU-Ebene dafür eintreten, dass Erzeugnisse, die besonders besorgniserregende Stoffe enthalten und freisetzen können, im Rahmen der bestehenden Chemikalienverordnung REACH (Registration, Evaluation, Authorisation and Restriction of Chemicals $)^{34}$ geregelt werden, wenn sie nicht in der EU hergestellt werden. Eine geeignete und bewährte Maßnahme zur Regelung von Stoffen in Erzeugnissen ist das REACH-Beschränkungsverfahren. Die Regulierung dieser Erzeugnisse soll beschleunigt und erleichtert werden." 35

26) Richtlinie (EU) 2018/851, ABl. 2018 L 150, S. 109.

27) Europäische Kommission, COM(2018) 29 final.

28) Richtlinie (EU) 2019/904, ABl. 2009 L 155, S. 1.

29) Bundesministerium für Umwelt, Naturschutz und nukleare Sicherheit, Unsere Politik für weniger Plastikmüll.

30) CDU, CSU und SPD, Koalitionsvertrag zwischen CDU, CSU und SPD

31) CDU, CSU und SPD, Koalitionsvertrag zwischen CDU, CSU und SPD, S. 137, Zn. 6472.

32) CDU, CSU und SPD, Koalitionsvertrag zwischen CDU, CSU und SPD, S. 137, Zn. 6444.

33) CDU, CSU und SPD, Koalitionsvertrag zwischen CDU, CSU und SPD, S. 138, Zn. 6588.

34) Verordnung (EG) Nr. 1907/2006 (REACH-Verordnung).

35) CDU, CSU und SPD, Koalitionsvertrag zwischen CDU, CSU und SPD, S. 140, Zn. 6621. 
Zum Stand der Umsetzung der am 1.1.2016 in Kraft getretenen UN Agenda 2030 gab es 2019 eine Große Anfrage einzelner Abgeordneter und der Fraktion BÜNDNIS 90/DIE GRUNEN, die von der Bundesregierung am 19.9.2019 beantwortet wurde. ${ }^{36}$ Es wird auf zahlreiche Maßnahmen verwiesen, namentlich u.a. ,, auf die am 2. Juli 2019 in Kraft getretene EU-Richtlinie über die Verringerung der Auswirkungen bestimmter Kunststoffprodukte auf die Umwelt (,Single Use Plastics“), auf das neue Verpackungsgesetz mit strengeren Recyclingvorgaben“" und ,auf den 5-Punkte-Plan des Bundesumweltministeriums für weniger Plastik und mehr Recycling." Weiterhin gebe es die vom Bundesministerium für Bildung und Forschung (BMBF) ins Leben gerufene Initiative „Plastik in der Umwelt - Quellen, Senken, Lösungsansätze“, mit der auf das Thema Plastik in der Umwelt bezogene Forschungsvorhaben unterstützt würden; das langfristige Ziel dieser Initiative sei es, „Kunststoffe in der Umwelt spürbar zu reduzieren ". ${ }^{37}$ Interessant an der Initiative des BMBF ist der interdisziplinäre Ansatz, so sollen sich z. B. zum Thema „Recycling und Produktentwicklung“ Experten aus den Sparten Recycling, Abfall- und Chemikalienrecht und Einsatz von Recyclingprodukten zusammenfinden ${ }^{38}$, um die vielfältigen Zusammenhänge zwischen der Produktentwicklung und einem guten Recyclingergebnis aufzuzeigen und Empfehlungen für eine Gesamtoptimierung zu erarbeiten.

\subsubsection{5-Punkte-Plan für weniger Plastik}

und mehr Recycling

Im November 2019 hat das Bundesministerium für Umwelt, Naturschutz und nukleare Sicherheit (BMU) seinen 5-Punkte-Plan für weniger Plastik und mehr Recycling vorgestellt. ${ }^{39}$ Die 5 Aktionspunkte lauten:

- Überflüssige Produkte und Verpackungen vermeiden

- Verpackungen und andere Produkte umweltfreundlicher gestalten

- Recycling stärken, mehr Rezyklate einsetzen

- Vermeidung von Kunststoffen in Bioabfällen

- Internationales Engagement gegen Meeresmüll und für einen nachhaltigen Umgang mit Kunststoffen

Flankiert wird diese Politik durch die Kampagne „Nein zur Wegwerfgesellschaft“. Insgesamt werden vom BMU aktuell bereits viele der aktuell drängendsten Problematiken angesprochen. Nicht ausreichend adressiert sind allerdings die Themen der gesundheitlichen Unbedenklichkeit von Plastikprodukten sowie der Plastikkontamination von Wasser, Böden und Luft.

\subsubsection{Initiativen von NGOs und privaten Organisationen}

Auch Nichtregierungsorganisationen (NGOs) und private Organisationen sind beim Thema Plastik aktiv. Stellvertretend für viele andere seien die Forderungen des World Wide Fund For Nature (WWF) mit seiner Kampagne „No Plastics in Nature" genannt. Es wird gefordert, dass bis Ende 2021 ein weltweit rechtsverbindliches Abkommen zur Beendigung der Kunststoffverschmutzung abzuschlieBen, sozusagen ein Pariser Abkommen für Kunststoffe. Dahinter steckt die Erkenntnis, dass das weltweite Kunststoffproblem mit der Klimakrise vergleichbar ist und ebenso wie diese ein weltweites Bewusstsein erfordert und konsequente und schnelle Lösungen unter Einbeziehung aller relevanten Akteure, Regierungen, Unternehmen und Öffentlichkeit, gefunden werden müssen. ${ }^{40}$

Zusammenfassend ist festzustellen, dass die Bundesregierung sich sehr deutlich zur Lösung der mit Kunststoffverpackungen und Kunststoff-Einwegprodukten zusammenhängenden Problemstellungen sowie zur Umsetzung der Agenda 2030 verpflichtet hat. Viele der Aktivitäten befinden sich noch im Anfangsstadium. Anders als beim alles überstrahlenden Thema Klima gibt es hier noch keinen ressortübergreifend integrierten Gesamtplan oder gar ein „Plastikkabinett“ (in Anspielung auf das „Klimakabinett ${ }^{41 \text { “" }}$ ein besonderer Ausschuss der Bundesregierung zur Umsetzung des Klimaschutzplans 2050). Der politische Wille ist klar erkennbar, Fokussierung und Integration sind sicher noch ausbaufähig.

\section{Vermeidung von Littering}

Ein großes Problem stellt der achtlose Umgang mit Kunststoffverpackungen und Einweg-Kunststoffprodukten nach ihrer meist sehr kurzen Nutzung dar. Diese Gegenstände werden nicht selten achtlos entsorgt und gelangen so in die Umwelt. Laut $\$ 28$ Abs. $1 \mathrm{KrWG}$ dürfen in Deutschland Abfälle zum Zweck der Beseitigung nur in den dafür zugelassenen Anlagen oder Einrichtungen (Abfallbeseitigungsanlagen) behandelt, gelagert oder abgelagert werden, was aber nicht der Realität entspricht.

\subsection{Straf- und bußgeldrechtlicher Rechtsrahmen}

Allein das Gebot zur ordnungsgemäßen Entsorgung reicht nicht aus, um eine Mülldisziplin zu gewährleisten. Erfahrungsgemäß führen Strafandrohungen in aller Regel zu regelkonformeren Verhaltensweisen, wobei allerdings zu beachten ist, dass die Strafandrohung zwar angemessen, aber auch spürbar sein muss, um nachweisbare Verhaltensänderungen herbeizuführen.

In Deutschland gibt es ein sehr umfangreiches Umweltstrafrecht. Gravierende Straftaten sind im Strafgesetzbuch, $\int \$ 324 \mathrm{ff}$. StGB, geregelt. Sofern es um die achtlose Entsorgung von Kunststoffverpackungen und EinwegKunststoffprodukten durch einzelne Verbraucher geht, handelt es sich in aller Regel jedoch nur um Ordnungswidrigkeiten, die von den Bundesländern im Rahmen von Bußgeldkatalogen geregelt sind. Gleichwohl hat das Littering in der Vergangenheit trotz der bestehenden Strafandrohungen erhebliche Ausmaße angenommen. So werden z. B. allein vom Landesbetrieb Straßen NRW jedes Jahr rund 16000 Tonnen Müll aufgesammelt und die Stadt Köln beziffert ihre jährlichen Aufwendungen für die Reinigung öffentlicher Flächen von wildem Müll auf 7,4 Mio. Euro. ${ }^{42}$ Offensichtlich waren die Strafandrohungen noch nicht ausreichend und/oder es gibt ein Überwachungsdefizit.

Hier setzen derzeit immer mehr Bundesländer an. In Nordrhein-Westfalen wurde z.B. 2019 ein neuer Bußgeldkatalog für den Bereich Abfall vom Umweltministerium veröffentlicht. ${ }^{43}$ Dieser gibt den zuständigen Behörden eine Entscheidungshilfe und einen Rahmen für die Festlegung von Verwarnungs- und Bußgeldern im Bereich des Abfallrechts. Dieser sieht u. a. eine Reihe von Verschärfungen vor mit dem Ziel, die acht- und rücksichtslose Müllentsorgung einzudämmen. So wird jetzt ein Bußgeld in Höhe von $100 €$ empfohlen, mit dem z.B. das Wegschnippen einer Zigarettenkippe oder die Entsorgung einer Burger-Verpackung am Straßenrand geahndet werden soll; zuvor lag die Empfehlung dafür bei nur 10 bis $25 €{ }^{44}$ Und auch andere

36) BT-Drs. 19/13352

37) BMBF, Plastik in der Umwelt, Ziele der Initiative.

38) BMBF, Plastik in der Umwelt, Querschnittsthemen.

39) BMU, Kampagne „Nein zur Wegwerfgesellschaft“ und 5-Punkte-Plan des Bundesumweltministeriums für weniger Plastik und mehr Recycling.

40) WWF, Plastics - What WWF is Doing.

41) ZDF, Bundesregierung setzt Klimakabinett ein.

42) MULNV Nordrhein-Westfalen, Bußgeldkatalog für den Bereich Abfall, Pressemitteilung vom 4.6.2019.

43) MULNV Nordrhein-Westfalen, Buß- und Verwarnungsgeldkatalog „Abfallrecht“, Stand Juni 2019.

44) MULNV Nordrhein-Westfalen, Bußgeldkatalog für den Bereich Abfall, Pressemitteilung vom 4.6.2019. 
Bundesländer wie z.B. Berlin sind aktuell dabei, ihre Verwarnungsgelder und Bußgelder zur Eindämmung des Litterings in ähnlicher Weise zu verschärfen. ${ }^{45}$

$\mathrm{Ob}$ diese Strafverschärfungen zu einem messbaren Rückgang des wilden Mülls führen werden, hängt sicher auch von der Intensität der Überwachung und der strikten Ahndung festgestellter Ordnungswidrigkeiten ab. Daneben kommt der Information und Aufklärung der Bevölkerung, z. B. mit Informationskampagnen im Kampf gegen das Littering, ebenfalls eine große Bedeutung zu.

\subsection{Gesetzliche Regelungen gegen Umweltverschmutzung durch exportierte Abfälle}

In Deutschland wird häufig auf die im internationalen Vergleich sehr hohe Verwertungsquote von rund $97 \%$ für Verpackungsabfälle im Rahmen der dualen Systeme verwiesen. Eine Besonderheit ist, dass die dualen Systeme in Deutschland sich die Menge der exportierten Kunststoffabfälle auf ihre Recyclingquoten anrechnen können, wenn diese zum Zweck des Recyclings in das Zielland verbracht werden. Es fallen jedoch auch erhebliche Mengen an Kunststoffabfällen außerhalb der dualen Systeme an, so dass das Exportthema nach wie vor relevant ist, denn immerhin lag Deutschland 2018 mit rund 701000 Tonnen Plastikmüll ${ }^{46}$ auf Rang 3 der weltweiten Plastikmüll-Exporteure.

Die Abfallverbringung ist international wie national umfangreich geregelt. Die Grundlagen dazu finden sich in der EU-Abfallverbringungsverordnung (AVV), die wiederum auf dem Basler Ubereinkommen ${ }^{47}$ und einem OECDRatsbeschluss aufbaut. ${ }^{48}$ Zudem gibt es für die OECDStaaten ein System für die Notifizierung, Identifizierung und Kontrolle der grenzüberschreitenden Verbringung von Abfällen zur Verwertung. In Deutschland enthält das Abfallverbringungsgesetz (AbfVerbrG) die darauf aufbauenden rechtlichen Regelungen. Es handelt sich um ein insgesamt sehr kompliziertes Rechtsgebiet, das noch eine Reihe weiterer relevanter Regelungen umfasst. ${ }^{49}$

Bedeutsam ist, dass bis heute viele Kunststoffabfallfraktionen derzeit noch unter die sog. grüne Liste ${ }^{50}$ fallen, d.h. sie sind als ungefährlich eingestuft und dürfen grundsätzlich ohne Genehmigung exportiert werden. Der Vorteil des Kunststoffmüllexports besteht darin, dass in den Niedriglohn-Zielländern auch minderwertige Stofffraktionen von Hand nachsortiert werden können, was hierzulande nicht mehr wirtschaftlich darstellbar ist. Der Nachteil ist, dass in den Zielländern wesentlich geringere Umweltstandards herrschen und es nicht kontrollierbar ist, wie dort mit den Sortierresten verfahren wird. Es ist dokumentiert und weiterhin zu befürchten, dass ein großer Anteil der Sortierreste unkontrolliert in die Umwelt gelangt oder schlecht deponiert bzw. in Gewässer gekippt wird.

Im Mai 2019 haben die 187 Vertragsstaaten des Basler Übereinkommens beschlossen, dass ab dem 1.1.2021 kontaminierte, gemischte oder nicht recyclingfähige Kunststoffabfälle auch zu den vorher genehmigungspflichtigen Exporten gehören sollen. Nach den neuen Vorgaben können nur noch sortenreine Abfälle und weitgehend störstofffreie Mischungen aus Polypropylen, Polyethylen und PET mit anderen Ländern frei gehandelt werden und diese müssen nachweislich zum Recycling bestimmt sein. Dieser Beschluss soll in einen rechtsverbindlichen OECD-Beschluss und anschließend in die europäische Verordnung über die Verbringung von Abfällen übernommen werden. ${ }^{51} \mathrm{Da}-$ mit besteht eine hohe Wahrscheinlichkeit, dass ab 2021 der Export von Kunststoffverpackungsmüll aus Deutschland deutlich zurückgehen könnte.

\subsection{Rechtsrahmen zur Vermeidung von Abfällen}

Die Vermeidung von Abfällen kann auf vielfältige Weisen geschehen. Vor allem die Wiederverwertung, Pfand- und
Nachfüllsysteme, aber auch die Vermeidung von Lebensmittelabfällen haben relevante Auswirkungen für die Müllvermeidung im Zusammenhang mit Kunststoffverpackungen und Einweg-Kunststoffprodukten.

Die wirksamste Maßnahme gegen aus Kunststoffverpackungen und Einweg-Kunststoffprodukten resultierenden Plastikmüll ist allerdings, wenn diese Gegenstände erst gar nicht produziert und in Verkehr gebracht werden. Dies kann u.a. dadurch gewährleistet werden, dass bestimmte Gegenstände verboten oder streng reglementiert werden, aber auch durch eine veränderte Verbrauchernachfrage, der in der Regel ein erhöhtes Problembewusstsein vorausgehen muss. Die Vermeidung ist - neben der Verwertung konstitutiv für die Kreislaufwirtschaft, $\$ 3$ Abs. 19 KrWG. ${ }^{52}$ Der Begriff der Vermeidung, wie er in $\$ 3$ Abs. $20 \mathrm{KrWG}$ eine Legaldefinition erhalten hat, zielt auf eine umfassende Betrachtung. Dabei geht es nicht nur um die Abfallmenge, sondern auch um die Verringerung der Schädlichkeit von Abfällen sowie um die Reduktion von ,schädlichen Stoffen in Materialien und Erzeugnissen". Zur Vermeidung zählen weiterhin die ,,abfallarme Produktgestaltung, die Wiederverwendung von Erzeugnissen oder die Verlängerung ihrer Lebensdauer sowie ein Konsumverhalten, das auf den Erwerb von abfall- und schadstoffarmen Produkten sowie die Nutzung von Mehrwegverpackungen gerichtet ist.“

$\int 6$ Abs. 1 KrWG bringt klar zum Ausdruck, dass Maßnahmen der Vermeidung im Rahmen der Abfallhierarchie die oberste Priorität einnehmen; Die Abfallvermeidung ist ein Ausdruck des Vorsorgeprinzips, eines der grundlegenden Umweltschutz-Prinzipien. Dieses besagt, dass umweltpolitische Maßnahmen so zu gestalten sind, dass Umweltgefahren vermieden und die natürlichen Ressourcen möglichst schonend in Anspruch genommen werden. Das heißt, dass nicht nur drohende Gefahren abzuwehren und bereits bestehende Schäden zu beseitigen sind. Es gilt vielmehr, von vornherein Entwicklungen zu verhindern, die zukünftig zu Umweltbelastungen führen können.

$\$ 7 \mathrm{KrWG}$ formuliert Grundpflichten der Kreislaufwirtschaft. Die Pflicht zur Abfallvermeidung gem. $\$ 7$ Abs. 1 KrWG nennt keinen speziellen Adressaten dieser Verpflichtung, sondern verweist auf $\$ 13 \mathrm{KrWG}$ und auf Rechtsverordnungen, die auf Grund der $\$ \$ 24$ und $25 \mathrm{KrWG}$ erlassen worden sind.

\$23 KrWG konkretisiert die Produktverantwortung, die sowohl die Entwickler, Hersteller, Verarbeiter als auch die Vertreiber von Erzeugnissen trifft. Danach ist bereits bei der Produktgestaltung anzusetzen, damit sowohl bei der Herstellung als auch beim Gebrauch das Entstehen von Abfällen vermieden wird. $\$ 23$ Abs. $2 \mathrm{KrWG}$ präzisiert diese Verpflichtung beispielhaft und benennt dabei Grundprinzipien der Kreislaufwirtschaft. In Bezug auf Kunststoffverpa-

45) Senatsverwaltung für Umwelt, Verkehr und Klimaschutz, Neuer Bußgeldkatalog für den Umweltschutz beschlossen, Pressemitteilung vom 24.10.2019.

46) Statista, Die größten Plastikmüll-Exporteure der Welt, Stand 20.2.2020, abrufbar unter https://de.statista.com/infografik/ 18340/die-groessten-plastikmuell-exporteure-der-welt/.

47) Basel Convention on the Control of Transboundary Movements of Hazardous Wastes and their Disposal, Stand 11.3.2020, abrufbar unter https://www.basel.int/Portals/4/download.aspx?d=UNEPCHW-IMPL- CONVTEXT.English.pdf.

48) Wuttke, Grenzüberschreitende Abfallverbringung (https://www. umweltbundesamt.de/sites/default/files/medien/421/dokumente/grenzueberschreitende_abfallverbringung_wuttke.pdf).

49) Wuttke, (Fn. 48).

50) Umweltbundesamt, Liste der Abfälle, die den allgemeinen Informationspflichten nach Art. 18 des Basler Abkommens unterliegen.

51) IHK Karlsruhe, Verschärfte Regeln für Export von Plastikabfällen und Handel mit alten Elektrogeräten.

52) Frenz, in: Urban/Halm (Hrsg.), UNIKAT-Fachtagung Abfallvermeidung, S. 30 
ckungen und Kunststoff-Einwegprodukte ist z. B. bedeutsam, dass Produkte möglichst mehrfach verwendbar und nach Gebrauch die Abfälle grundsätzlich ordnungsgemäß, schadlos und hochwertig zu verwerten oder umweltverträglich zu beseitigen sein sollen, $\$ 23$ Abs. 2 Nr. 1 KrWG. Bei der Herstellung sollen vorrangig verwertbare Abfälle oder sekundäre Rohstoffe (z.B. Rezyklate) verwendet werden, $\$ 23$ Abs. 2 Nr. 2 KrWG. Konkrete Pflichten begründet aber auch dies noch nicht, diese ergiben sich gem. $\$ 23$ Abs. 4 KrWG erst aus den von der Bundesregierung auf Grund der \$\$ 23 und $24 \mathrm{KrWG}$ erlassenen Rechtsverordnungen. Ein wichtiges Beispiel für eine auf dieser Grundlage erlassene Rechtsverordnung war die Verpackungsverordnung, die am 1.1.2019 durch das Verpackungsgesetz abgelöst wurde.

Auch im Verpackungsgesetz findet sich der Grundsatz der Vermeidung wieder, wie er zuvor auch schon in $\$ 12$ VerpackV enthalten war. So heißt es in $\$ 4$ Nr. 1 VerpackG, dass Verpackungsvolumen und -masse auf das Mindestmaß $\mathrm{zu}$ begrenzen sind.

Gerade in den letzten Jahren wurde eine Reihe von Maßnahmen zur Abfallvermeidung auf den Weg gebracht. Das BMU hat 2013 das „Abfallvermeidungsprogramm des Bundes unter Beteiligung der Länder" herausgegeben. ${ }^{53} \mathrm{Da}$ mit wurde auch die Verpflichtung aus der EU-Richtlinie 2008/98/EG umgesetzt, nach dessen Art. 29 Abs. 1 alle Mitgliedsländer bis zum 12.12.2013 Abfallvermeidungsprogramme aufzustellen hatten. In dem umfassenden Dokument finden sich keine direkten Hinweise auf Kunststoffe oder Plastik, die Hinweise sollen sich vielmehr grundsätzlich auf verschiedene Stoffströme anwenden lassen. ${ }^{54} \mathrm{Als}$ konkrete Maßnahmen zur Abfallvermeidung wird u.a. empfohlen, die verschiedenen Akteure und Bevölkerungsgruppen in Bezug auf die Notwendigkeit und Möglichkeiten der Abfallvermeidung zu sensibilisieren, eine Intensivierung von Forschung und Entwicklung mit den Zielen abfallvermeidendes Produktdesign und abfallarme Produktion; außerdem wird empfohlen, die Rechtsetzung und den Vollzug stärker auf die Förderung der Abfallvermeidung zu fokussieren. ${ }^{55}$ Zudem wird die Vermeidung von Lebensmittelabfällen als weitere Zielrichtung genannt ${ }^{56}$, was ebenfalls eine erhebliche Bedeutung im Kontext der Kunststoffverpackungen und der Kunststoff-Verpackungsabfälle hat. Insgesamt wirkt das Abfallvermeidungsprogramm von 2013 allerdings $\mathrm{zu}$ unkonkret und ohne wirklich messbare Zielgrößen, z. B. für die Verringerung der Menge der Gewerbeund Siedlungsabfälle pro Einwohner, eine Einschätzung, die auch von Umweltorganisationen artikuliert wurde. ${ }^{57}$

Das Thema „Vermeidung" ist somit rechtlich in vielen Ausprägungen verankert. Allerdings dürfen eigentlich vermeidbare Kunststoffverpackung immer noch in Verkehr gebracht werden und es gibt dafür auch keine Sanktionsmöglichkeiten. Seit Jahren steigen in Deutschland die Mengen an Kunststoffabfällen, es besteht dringender Handlungsbedarf. Die aktuelleren Impulse und die stärkere Betrachtung des Themas Vermeidung kommen derzeit eindeutig aus der EU.

\section{Braucht Deutschland ein Kunststoffgesetz?}

\subsection{Rechtsrahmen für das "Inverkehrbringen“"}

Wer in Deutschland Kunststoffverpackungen oder Kunststoff-Einwegprodukte in Verkehr bringt, hat eine Vielzahl von Vorschriften aus verschiedenen Rechtsgebieten zu beachten. Die wichtigsten $\mathrm{zu}$ beachtenden rechtlichen Vorschriften stammen aus den Bereichen Chemikalienrecht (einschließlich Regelungen für Materialien und Gegenstände aus Kunststoff mit Lebensmittelkontakt) und dem Abfallrecht.

\subsubsection{Chemikalienrecht}

Zunächst müssen die Vorschriften des Chemikalienrechts beachtet werden, das überwiegend auf EU-Verordnungen basiert. Die zentrale Vorschrift im Chemikalienrecht ist die REACH-Verordnung ${ }^{58}$, die in allen EU-Mitgliedsstaaten unmittelbar gilt. Sie regelt Registrierungs- und $\mathrm{Zu}-$ lassungspflichten, Beschränkungen, aber auch Kommunikationspflichten in der Lieferkette (Sicherheitsdatenblatt). Ergänzt wird die REACH-Verordnung für bestimmte Anwendungsbereiche durch zusätzliche Regelungen, wie z.B. die CLP-Verordnung (Classification, Labelling and $\mathrm{Pa}-$ ckaging ${ }^{59}$, die die Einstufung, Kennzeichnung und Verpackung von gefährlichen Stoffen und Gemischen regelt und die POP-Verordnung (persistent organic pollutants) ${ }^{60}$ über persistente organische Schadstoffe. In Deutschland gibt es zusätzlich das Chemikaliengesetz, das in erster Linie die europäischen Regelungen umsetzt und um weitere nationale Vorgaben ergänzt.

Die europäische Chemikalienagentur (ECHA, European Chemicals Agency) hat 2017 neue Guidelines veröffentlicht und darin klargestellt, dass auch Verpackungen eigene Artikel darstellen und somit den gleichen Informationsanforderungen nach der REACH-Verordnung unterliegen wie andere Artikel auch. ${ }^{61}$ Es drängt sich aber angesichts der Vielzahl der in Verkehr gebrachten Kunststoffverpackungen und Einweg-Kunststoffartikel allerdings die Frage auf, ob die vielfältigen und komplizierten Regelungen des Chemikalienrechts in der Praxis durchgängig angewandt werden und wie es um die deren Beachtung bei den vielen importierten Waren steht. Dazu stellte das Umweltbundesamt 2018 in einer Ausarbeitung ${ }^{62}$ u.a. fest, dass REACH zwar die Informationslage über Chemikalien wesentlich verbessert und sich dadurch auch das Bewusstsein über Chemikalienrisiken sich insgesamt erhöht habe, dennoch seien die REACH-Pflichten teilweise nicht ausreichend bekannt. Es müssten Regelungen für zulassungspflichtige, besonders besorgniserregend Stoffe (SVHC, Substance of Very High Concern) in importierten Erzeugnissen gefunden werden. Die Meldung nach REACH Artikel 7 (2) und die Kommunikation über SVHC in Erzeugnissen in der Lieferkette und an die Verbraucher funktioniere nur unzureichend. ${ }^{63}$ Für die Überwachung der Verordnung ständen zu wenige Ressourcen zur Verfügung und es gebe einen Bedarf an (weiterer) Harmonisierung. Diese Aussagen lassen den Schluss zu, dass es zwar ein sehr strenges Chemikalienrecht gibt, dass aber die vollständige Durchsetzung und Überwachung in der Praxis erhebliche Schwierigkeiten bereitet.

Es gibt eine große Anzahl wissenschaftlicher Untersuchungen zum Thema der von den chemischen Inhaltsstoffen in Kunststoffprodukten ausgehenden Gesundheitsund/oder Umweltgefahren. Zudem gibt es eine Reihe von heute noch zugelassenen chemischen Stoffen, die ein Recycling erschweren oder sogar unmöglich machen. Daher kann das Chemikalienrecht als ein besonders wichtiger Schlüssel zur Verbesserung der gesundheitlichen Unbedenklichkeit, der Umweltverträglichkeit, der Wiederver-

53) BMU, Abfallvermeidungsprogramm des Bundes unter Beteiligung der Länder, Juli 2013

54) BMU, (Fn. 53), S. 37.

55) BMU, (Fn. 53), S. 26.

56) BMU, (Fn. 53), S. 29.

57) Bund für Umwelt und Naturschutz Deutschland e.V. (Hrsg.), Stellungnahme zum Abfallvermeidungsprogramm des Bundes unter Beteiligung der Länder.

58) Verordnung (EG) Nr. 1907/2006, ABl. 2006 L 396, S. 1

59) Verordnung (EG) Nr. 1272/2008, ABl. 2008 L 353, S. 1

60) Verordnung (EG) Nr. 850/2004, ABl. 2004 L 158, S. 7 ff.

61) QZ-Online, Portal für Qualitätsmanagement, Auch Verpackungen müssen REACH-konform sein.

62) Umweltbundesamt, REACH-Weiterentwicklung Vergleich des Review-Berichts der EU-Kommission mit verschiedenen Studien und Berichten im Kontext der REACH-Überprüfung, UBA-Texte 93/2018.

63) Umweltbundesamt, (Fn. 62), S. 10. 
wertbarkeit und auch der Recyclingfähigkeit angesehen werden. Hier bestehen große politische, rechtliche und technische Handlungsbedarfe, die von den wesentlichen Akteuren aufgegriffen werden müssen. Von Seiten der politisch Verantwortlichen muss es hierzu klare Ziel- und Strategievorgaben geben.

\subsubsection{Abfallrecht}

Die Produktverantwortung ist ein zentrales Prinzip der Kreislaufwirtschaft, worin das Verursacherprinzip zum Ausdruck kommt. Zur Produktverantwortung gehört nach dem deutschen Abfallrecht auch die Entsorgungsverantwortung $^{64}$, die bereits beim Inverkehrbringen zu beachten ist. Diese ist sowohl im Kreislaufwirtschaftsgesetz als auch im Verpackungsgesetz verankert.

\subsubsection{Novellierung des Kreislaufwirtschaftsgesetzes}

Aktuell befindet sich der ,Entwurf eines Gesetzes zur Umsetzung der Abfallrahmenrichtlinie der Europäischen Union" (Bearbeitungsstand: 3. 2.2020) im Gesetzgebungsverfahren. ${ }^{65}$ Dieser beinhaltet eine Novellierung des Kreislaufwirtschaftsgesetzes. Im Folgenden wird nur auf die Punkte eingegangen, die im Hinblick auf Kunststoffverpackungen, Einwegkunststoffartikel und Kunststoffabfälle von Bedeutung sind.

Zunächst wird in einem neuen $\$ 3$ Abs. 5a KrWG-Entwurf (KrWG-E) klargestellt, was Siedlungsabfälle sind. Dieses sind gemischt und getrennt gesammelte Abfälle, zu denen nach Nr. 1 der Vorschrift auch Kunststoff aus privaten Haushaltungen gehört. Diese neue Definition von Siedlungsabfällen wiederum ist wichtig für die beabsichtigten Anderungen des $\$ 14 \mathrm{KrWG}$. Während die aktuelle Fassung des $\$ 14$ KrWG sich auf das Recycling und die stoffliche Verwertung von Papier-, Metall-, Kunststoffund Glasabfällen bezog und darauf bezogen bereits ab dem 1. Januar 2020 eine Quote von 65 Gewichtsprozent für die Vorbereitung zur Wiederverwendung und das Recycling vorsieht, soll sich die Neufassung auf den weiteren Begriff der Siedlungsabfälle beziehen, für die ab dem 1. Januar 2020 lediglich eine Quote von 50 Gewichtsprozent gelten soll, die in gestaffelten Schritten auf eine Quote von 65 Gewichtsprozent angehoben werden soll, die spätestens am 1. Januar 2035 erreicht sein soll. Somit würde auch der Anteil der Kunststoffabfälle in den neuen Gesamtquoten für Siedlungsabfälle aufgehen, so dass daraus keine spezifische Recyclingquote für Kunststoffabfälle abzuleiten wäre. Es verwundert nicht, dass der geringere und spätere Ansatz der neuen Recyclingquoten teilweise als zu wenig ambitioniert kritisiert wird. ${ }^{66}$

$\S 5$ Abs. $1 \mathrm{KrWG}-\mathrm{E}$ sieht vor, dass für einen Stoff oder Gegenstand auch dann die Abfalleigenschaft endet, wenn dieser ein Recyclingverfahren durchlaufen hat und er die weiteren in Nr. 1 bis Nr. 4 genannten Voraussetzungen erfüllt. Diese Vorschrift ist auch für das Kunststoffrecycling von Bedeutung, denn danach müssen auch Rezyklate alle geltenden technischen Anforderungen, Rechtsvorschriften und Normen erfüllen und ihre Verwendung darf insgesamt nicht zu schädlichen Auswirkungen auf Mensch oder Umwelt führen. Somit werden auch die stoffliche Qualität von Rezyklaten und vor allem die Abwesenheit bzw. die noch tolerierte Menge an Schadstoffen zu entscheidenden Faktoren für die Beendigung der Abfalleigenschaft. $\ 5$ Abs. 2 KrWG-E sieht eine weitreichende Ermächtigung der Bundesregierung vor, per Rechtsverordnung mit Zustimmung des Bundesrates Regelungen für das Ende der Abfalleigenschaft zu treffen. Diese sollen ein hohes Maß an Schutz für Mensch und Umwelt sicherstellen und zur Ressourcenschonung beitragen. Dies könnte z. B. auch dazu führen, dass per Rechtsverordnung die für das Kunststoffrecycling zulässigen Behandlungsverfahren und -methoden sowie Qualitätskriterien mit Schadstoffgrenzwerten festgelegt werden könnten.
Auch $₫ 9$ Abs. 2 KrWG-E könnte für Kunststoffabfälle relevant werden. Nach der Neufassung ist eine energetische Verwertung von mit dem Ziel der Wiederverwendung oder zum Recycling getrennt gesammelten Abfällen nur noch für die nach der Behandlung (z. B. Sortierung) verbleibenden Reste zulässig. Auch diese Restabfallfraktionen sollen grundsätzlich nur dann energetisch verwertet werden dürfen, wenn auch dabei der Schutz von Mensch und Umwelt mindestens in gleicher Weise wie durch die Vorbereitung zur Wiederverwendung oder das Recycling gewährleistet ist. Von dieser Vorschrift könnte eine deutliche Anreizwirkung zur weiteren Verbesserung der Sortierergebnisse ausgehen.

$\$ 20$ Abs. 2 KrWG-E legt fest, dass öffentlich-rechtliche Entsorgungsträger zur getrennten Sammlung einiger $\mathrm{Ab}$ fallfraktionen verpflichtet sind, u.a. gem. Nr. 2 auch für Kunststoffabfälle.

Sehr wichtige Neuregelungen zur Produktverantwortung enthält $\$ 23 \mathrm{KrWG}-\mathrm{E}$. Das Ziel der Produktverantwortung ist es, möglichst dem Entstehen von Abfällen bereits bei der Produktion vorzubeugen. Bei der Fortentwicklung der Produktverantwortung fließt sehr erheblich das neue Unionsrecht ein, insbesondere die durch Artikel 8 AbfRRL ${ }^{67}$ vorgegebene erweiterte Herstellerverantwortung. Im Hinblick auf Kunststoffe sind einige der angedachten Änderungen relevant. $\$ 23$ Abs. 2 Nr. 1 KrWG-E legt, wie schon die aktuelle Fassung, die besondere Bedeutung des Produktdesigns fest, u.a. im Hinblick die ordnungsgemäße, schadlose und hochwertige Verwertung sowie die umweltverträgliche Beseitigung. Weitergehend regelt $\$ 23$ Abs. 2 Nr. 2 KrWG-E, dass zur Produktverantwortung auch der vorrangige Einsatz von sekundären Rohstoffen, insbesondere Rezyklaten, bei der Herstellung von Erzeugnissen gehören soll. Nr. 5 erwähnt als Inhalt der Produktverantwortung ausdrücklich die Senkung des Gehalts an gefährlichen Stoffen sowie die Kennzeichnung von schadstoffhaltigen Erzeugnissen; hier hängt die Relevanz für Kunststoffprodukte allerdings von der Einstufung der Gefährlichkeit der chemischen Inhaltsstoffe ab. Nr. 6 erweitert die Hinweispflichten im Hinblick auf die Beseitigungsmöglichkeiten. Nr. 9 legt eine umfassende Informations- und Beratungspflicht gegenüber der Öffentlichkeit fest. Es sollen Produkte nicht nur zu den Verwertungsoptionen gekennzeichnet werden, vielmehr ,,ist die Öffentlichkeit auch über die Möglichkeiten der Vermeidung sowie Maßnahmen zur Verhinderung der Vermüllung der

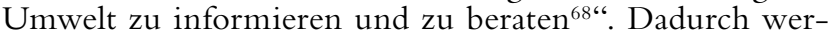
den bei der Abfallberatung erstmals auch die Hersteller und Vertreiber als zentrale Akteure der Abfallwirtschaft in die Pflicht genommen. Nr. 10 enthält eine weitere Neuerung, nämlich die verursachergerechte Beteiligung von Herstellern und Vertreibern an den Kosten, die den öffentlichrechtlichen Entsorgungsträgern und sonstigen öffentlichrechtlich Verpflichteten für die Säuberung der Umwelt entstehen. Für Verpackungen ist dies bereits in $\$ 22 \mathrm{Ab}-$ satz 9 VerpackG angelegt. Nr. 10 entwickelt diesen Ansatz im Sinne der Einweg-Kunststoffrichtlinie fort.

64) Cancik, in: Schmehl/Klement (Hrsg.), GK-KrWG, Einleitung Rdnr. 62

65) Stand 14.7.2020, abrufbar unter http://www.bmu.de/gesetz/ gesetzesentwurf-eines-gesetzes-zur-umsetzung-der-abfallrahmenrichtlinie-der-europaeischen-union.

66) Bund für Umwelt und Naturschutz - BUND, Stellungnahme zum Referentenentwurf der Novellierung des „,Gesetz zur Förderung der Kreislaufwirtschaft und Sicherung der umweltverträglichen Bewirtschaftung von Abfällen (Kreislaufwirtschaftsgesetz KrWG)“, S. 6.

67) Richtlinie 2006/12/EG vom 5. 4. 2006, ABl. 2006 L 194, S. 47.

68) BMU, Referentenentwurf eines Gesetzes zur Umsetzung der Abfallrahmenrichtlinie der Europäischen Union (Stand: 5.8.2019), S. 65 . 
Die $\int \$ 24$ und $25 \mathrm{KrWG}-\mathrm{E}$ enthalten sehr umfangreiche und weitgehend Verordnungsermächtigungen für die Bundesregierung, wonach diese im Wege der Rechtsverordnungen die Anforderungen nach $\$ 23 \mathrm{KrWG}-\mathrm{E}$ näher bestimmen kann.

$§ 33 \mathrm{KrWG}-\mathrm{E}$ sieht deutliche Fortentwicklungen zu den bereits besprochenen Abfallvermeidungsprogrammen des Bundes unter Beteiligung der Länder vor. $\ 33$ Abs. 3 Nr. 2 KrWG-E macht sehr umfangreiche Vorgaben für die darzustellenden Abfallvermeidungsmaßnahmen. Relevant für die Kunststoffthematik sind insbesondere die Maßnahmen im Hinblick auf die Förderung und Unterstützung nachhaltiger Produktions- und Konsummodelle (Nr. 2a), die Verringerung der Verschwendung von Lebensmitteln (Nr. 2 g), die Förderung von Lebensmittelspenden und anderen Formen der Umverteilung von Lebensmitteln für den menschlichen Verzehr (Nr. 2h), die Förderung der Senkung des Gehalts an gefährlichen Stoffen in Materialien und Produkten (Nr. 2i), die Ermittlung von Produkten, die Hauptquellen der Vermüllung insbesondere der Natur und der Meeresumwelt sind und die Durchführung geeigneter Maßnahmen zur Vermeidung und Reduzierung des durch diese Produkte verursachten Müllaufkommens (Nr. 2k) sowie die Entwicklung und Unterstützung von Informationskampagnen, die für Abfallvermeidung und Vermüllung sensibilisieren (Nr. $2 \mathrm{~m}$ ).

\46 Abs. 2 und 3 KrWG-E regeln eine im Vergleich zur bisherigen Regelung wesentlich konkretere Abfallberatungspflicht der öffentlich-rechtlichen Entsorgungsträger, um künftig bessere Ergebnisse bei der ordnungsgemäßen Abfalltrennung zu erreichen.

Zusammenfassend ist festzustellen, dass der Gesetzentwurf eine Reihe von Regelungen enthält, die für die Kunststoffthematik relevant sind und bei korrekter Umsetzung auch zu sehr deutlichen Verbesserungen führen können. Wichtig wird sein, mit welchen Inhalten die Bundesregierung von ihren Verordnungsermächtigungen Gebrauch machen wird und wie ambitioniert diese ausgestaltet sein werden.

\subsubsection{Verpackungsgesetz}

Für Kunststoffverpackungen ist u.a. das Verpackungsgesetz (VerpackG) einschlägig, das gem. \$2 Abs. 1 VerpackG für alle Verpackungen gilt. Über die Vorgaben des Gesetzes soll erreicht werden, dass Verpackungsabfälle vorrangig möglichst vermieden bzw. einer Vorbereitung zur Wiederverwendung oder dem Recycling zugeführt werden und zusätzliche Wertstoffe für ein hochwertiges Recycling gewonnen werden. Im Wesentlichen soll dies durch eine Systembeteiligungspflicht bzw. die flächendeckende Einrichtung eines Erfassungs- und Verwertungssystems für private Haushaltungen erreicht werden. ${ }^{69}$

Gem. \$3 Abs. 8 VerpackG sind systembeteiligungspflichtige Verpackungen ,,mit Ware befüllte Verkaufs- und Umverpackungen, die nach Gebrauch typischerweise beim privaten Endverbraucher als Abfall anfallen“. Die Systembeteiligungspflicht soll eine umweltökonomische Steuerungswirkung entfalten, indem über die Pflicht zur Tragung der Entsorgungskosten durch die Verursacher ein starker Anreizeffekt zur Vermeidung von Verpackungsabfällen gesetzt werden soll. ${ }^{70}$ Die gesetzliche Grundverpflichtung für jeden, der eine neue, systembeteiligungspflichtige Verpackung in den Verkehr bringt, besteht darin, diese bei einem Anbieter des sogenannten Dualen Systems zu lizensieren. In $₫ 3$ Abs. 9 Verpack $G$ wird Inverkehrbringen als ,jede entgeltliche oder unentgeltliche Abgabe an Dritte im Geltungsbereich dieses Gesetzes mit dem Ziel des Vertriebs, des Verbrauchs oder der Verwendung“" definiert. Das Inverkehrbringen erfasst somit nicht nur die Abgabe der verpackten Ware an den Endverbraucher, sondern die Abgabe an jeden im Wirtschaftsverkehr tätigen Dritten. Auf jeder Vertriebsstufe liegt damit ein neuerliches Inver- kehrbringen vor. Dabei spielt es keine Rolle, ob die Abgabe gewerblich oder entgeltlich erfolgt; notwendig ist lediglich, dass die Abgabe mit dem Ziel des Vertriebs, des Verkaufs oder der Verwendung erfolgt. ${ }^{71}$ Das Ziel der Verpackungsverordnung von 1991 (VerpackV), dem Vorläufer des VerpackG, war es, die Inverkehrbringer von Verpackungen an den Kosten für deren Sammlung und Aufbereitung unmittelbar zu beteiligen (Duales System). Dadurch sollten Anreize für eine Reduzierung von Verpackungsmaterial gesetzt werden. Die Beteiligung erfolgte, indem sich die Hersteller von systembeteiligungspflichtigen Verpackungen mit diesen Verpackungen an einem Dualen System beteiligen oder einen Dritten damit beauftragen. ${ }^{72}$

In der Praxis zeigte das System der VerpackV jedoch deutliche Mängel, denn die Lizenzierung wurde bei weitem nicht von allen dazu verpflichteten Unternehmen durchgeführt. Die Systeme des dualen Entsorgungssystems nutzten für die Sammlung eine gemeinsame Infrastruktur, deren Kosten nach den Marktanteilen der an allen Systemen beteiligten Verpackungen aufgeteilt wurden. Es bestand somit für die Systeme der Anreiz, den eigenen Marktanteil als möglichst gering darzustellen. Die Branche war teilweise sehr kreativ, so wurden z. B. gröBere Mengen Verkaufsverpackungen der Kunden als selbst zurückgenommen oder als einer sogenannten Branchenlösung zugehörig definiert. Auch falsche und unzulängliche Angaben der Systembetreiber waren nicht unüblich. Dieses Verhalten wurde zudem dadurch begünstigt, dass der Vollzug der Vorschriften in die Zuständigkeit von sechzehn unkoordiniert agierenden Landesbehörden fiel, so dass ein wirksamer, flächendeckender und einheitlicher Vollzug nicht sichergestellt werden konnte. ${ }^{73}$ In der Folge waren die tatsächlichen Recyclingquoten - vor allem von Kunststoffen - deutlich niedriger als mit der Regelung bezweckt.

Mit dem neuen Verpackungsgesetz hat der Gesetzgeber beim Thema Verpackungslizenzierung gegenüber der VerpackV an entscheidenden Wirkpunkten nachgebessert. Das Ziel war es, einen funktionierenden Wettbewerb sowie ein gesetzeskonformes Verhalten aller Marktteilnehmer sicherzustellen. Dies soll u. a. dadurch bewirkt werden, dass bei Verstößen gegen die Verpflichtung zur Verpackungslizensierung - wie auch schon nach der VerpackV - Bußgelder verhängt werden können, \$34 VerpackG. Unter der Geltung der VerpackV war jedoch die Gefahr gering, im Falle von Verstößen tatsächlich erwischt und belangt zu werden. Da jetzt im Verpackungsgesetz im Abschnitt 5 (\$S 24-30 VerpackG) die Einführung der Zentralen Stelle Verpackungsregister geregelt ist, sind die realen Kontrollmöglichkeiten deutlich erweitert worden.

$\$ 4$ VerpackG definiert allgemeine Anforderungen an Verpackungen, die von Herstellern und Vertreibern zu beachten sind. Diese sind im Kontext mit den in $\$ 23 \mathrm{KrWG}$ zum Ausdruck gebrachten Zielsetzungen für Verpackungen im Hinblick auf Vermeidung, Wiederverwendung, Recycling, Verwertung bzw. schadstoffarme Beseitigung zu sehen. Es geht im Kern um eine abfallrechtliche Produktverantwortung, die bereits am Beginn der Prozesskette bei der Produktgestaltung ansetzt. ${ }^{74}$ Ein interessanter Wirkzusammenhang besteht mit $\$ 21$ VerpackG, denn

69) Kempkes, in: Schmehl/Klement (Hrsg.), GK-KrWG, $\$ 21$ VerpackG, Rdnr. 2

70) Flanderka et al., Verpackungsverordnung, $\mathbb{\$} 1$, Rdnr. 11

71) Bartholmes, in: Schmehl/Klement (Hrsg.), GK-KrWG, $\$ 3$ VerpackG, Rdnr. 33.

72) Eko-punkt GmbH, Neues Verpackungsgesetz (ehemals Verpackungsverordnung) im dualen System.

73) Ludewig, in: Schmehl/Klement (Hrsg.), GK-KrWG, $\$ 24$ VerpackG, Rdnr. 1.

74) Prelle, in: Schmehl/Klement (Hrsg.), GK-KrWG, $\$ 4$ VerpackG, Rdnr. 2. 
die Höhe der dort geregelten sog. Beteiligungsentgelte soll sich nach ökologischen Kriterien richten. \4 Abs. 1 VerpackG gibt zunächst vor, dass Verpackungsvolumen und -masse auf das Mindestmaß begrenzt werden sollen. Allerdings findet sich bereits im 2. Halbsatz die Einschränkung, dass dies nur unter dem Vorbehalt der Angemessenheit gilt, und zwar in Bezug auf die erforderliche Sicherheit und Hygiene sowie der Verbraucherakzeptanz. Diese Einschränkung führt zu der Frage, ob angesichts dieser sehr unbestimmten Beschreibung überhaupt das erforderliche Mindestmaß einer Verpackung sicher bestimmt werden kann, wenn sich die Hersteller auf diese Einschränkungen berufen. Die Möglichkeiten der Durchsetzung dürften für eine Behörde beschränkt sein, zumal es für den Tatbestand der übermäßigen Verpackung keinen Sanktionstatbestand in $\$ 34$ VerpackG gibt. Zudem geben Unternehmen die Beteilungsentgelte regelmäßig über ihre Preiskalkulation an die Verbraucher weiter, so dass abzuwarten bleibt, ob von $\$ 21$ VerpackG tatsächlich die gewünschte Lenkungswirkung in Richtung eines umweltgerechteren Verpackungsdesigns ausgeht. ${ }^{75}$ In den vergangenen Jahren war unter der bis Ende 2018 geltenden VerpackV jedenfalls ein stetiges Anwachsen der Abfallmenge aus Kunststoffverpackungen $\mathrm{zu}$ verzeichnen und es bleibt abzuwarten, wie sich die Abfallmenge unter der Geltung des Verpackungsgesetzes entwickeln wird.

\subsubsection{Fazit zum Rechtsrahmen für das "Inverkehrbringen“}

Es gibt eindeutige Handlungsbedarfe. Insbesondere das Chemikalienrecht einschließlich REACH muss an die Forderungen angepasst werden, dass Kunststoffverpackungen von der stofflichen Zusammensetzung so gestaltet sein müssen, dass diese gesundheitlich unbedenklich und umweltverträglich sind und die Wiederverwendung sowie das Recycling fördern, was eine weitgehende Schadstoffreduzierung voraussetzt.

Im Abfallrecht liegt mit dem KrWG und dem VerpackG ein grundsätzlich guter Rechtsrahmen vor. Der Gesetzentwurf zur Novelle des KrWG sieht vielfältige und zu begrüßende Neuerungen im KrWG vor. Es ist wichtig, dass dieser Entwurf bis zur Gesetzesverabschiedung nicht in wesentlichen Punkten noch abgeschwächt wird und dass die Bundesregierung anschließend von ihren weitreichenden Verordnungsermächtigungen ambitioniert Gebrauch macht.

Das VerpackG hat viele Schwächen der VerpackV beseitigt und beginnt, im Sinne des Gesetzes Wirkungen zu entfalten. Im Hinblick auf ein möglichst ökologisches Verpackungsdesign sollte noch ein Instrumentarium entwickelt werden, das beim Inverkehrbringen nicht nur auf die Lenkungswirkung der Beteiligungsentgelte setzt, sondern striktere Möglichkeiten zur Vermeidung des Inverkehrbringens für solche Verpackungen enthält, die die Grundforderungen (gesundheitlich unbedenklich, umweltverträglich, gut geeignet für Wiederverwendung, Recycling oder Kompostierung) nicht erfüllen. Das Verpackungsdesign sollte als wirksames Regelungsobjekt in den Fokus gerückt werden.

\subsection{Rechtsrahmen ,Wiederverwendung“}

Gem. \6 Abs. 1 KrWG stellt die Vorbereitung zur Wiederverwendung eine eigenständige Hierarchiestufe dar, die in der Priorität nach der Vermeidung an zweiter Rangfolge steht. Zudem gibt es in Deutschland eine Reihe von Mehrweg-Pfandsystemen, z.B. auch für Mehrweg-PETGetränkeflaschen, die seit Jahren etabliert sind. Allerdings muss auf die seit Jahren sinkende Mehrwegquote hingewiesen werden, die 2004 noch bei 66,3\% ${ }^{76}$ lag und im Jahr 2017 nur noch 43,6\% betrug ${ }^{77}$. Damit liegt die Mehrwegquote derzeit deutlich unter der gem. \$1 Abs. 3 VerpackG ausgegebenen Zielgröße von $70 \%$ für Mehrweg-Getränkever- packungen. An die Verfehlung dieser Zielgröße sind nach dem Verpackungsgesetz keine Sanktionen geknüpft. Die Tatsache, dass die Mehrwegquote vor Jahren bereits in der Nähe der $70 \%$-Zielmarke gelegen hatte, zeigt zum einen, dass die Erreichung grundsätzlich möglich wäre, zum anderen aber auch, dass die Politik einen verbindlichen Gesetzesrahmen vorgeben müsste, um die Marktteilnehmer zu einer Erhöhung der Mehrweganteile zu bewegen.

Insgesamt ist festzustellen, dass das Thema Wiederverwendung/Reuse bei Kunststoffverpackungen bislang lediglich bei PET-Mehrwegflaschen auch mengenmäßig eine wichtige Rolle spielt. Gesetzlich ist dieses Thema noch nicht verstärkt in den Fokus gerückt und die gesetzlichen Vorgaben erscheinen noch nicht als hinreichend konkret.

Der Rechtsrahmen „Wiederverwendung“ hält somit noch kein ausreichendes Instrumentarium zur Sicherstellung der gesetzlich geforderten Mehrwegquoten bereit. Hier sollte eine stärkere gesetzliche Verpflichtung verankert werden, um die Marktteilnehmer zu einer Trendwende zu bewegen.

\subsection{Rechtsrahmen „Recycling“}

Das Thema Recycling ist ein zentrales Element aller $\mathrm{Zu}-$ kunftsstrategien. Recycling steht gem. \6 Abs. 1 Nr. 3 KrWG auf der dritten Stufe der Abfallhierarchie, ohne Recycling kann keine Kreislaufwirtschaft funktionieren. Definiert ist der Begriff in $\$ 3$ Abs. $25 \mathrm{KrWG}$. Zur Förderung eines ordnungsgemäßen, schadlosen und hochwertigen Recyclings sind gem. $₫ 14$ Abs. $1 \mathrm{KrWG}$ u.a. Kunststoffabfälle getrennt $\mathrm{zu}$ sammeln, soweit dies technisch möglich und wirtschaftlich zumutbar ist. Ein Musterbeispiel sind PET-Einwegflaschen mit Pfand, die wegen der hohen Rücklaufquote tatsächlich zu 98,8\% recycelt werden. ${ }^{78}$

$\$ 16$ Abs. 2 S. 2 und 3 VerpackG sehen vor, dass die durch die Systeme erfassten Kunststoffe zu mindestens 90\% einer Verwertung zuzuführen sind und dass dabei mindestens 65 Prozent und ab dem 1.1.2022 $70 \%$ dieser Verwertungsquote durch werkstoffliche Verwertung (siehe $\$ 3$ Abs. 19 VerpackG) sicherzustellen sind. Da Recycling die typische Voraussetzung für eine werkstoffliche Verwertung ist, erzeugt dies für die Systeme einen hohen Druck in Richtung einer weiteren Ausweitung des Recyclings. Ergänzt wird diese Vorgabe durch eine weitere in $\$ 16$ Abs. 4 VerpackG, wonach die Systeme verpflichtet sind, ,im Jahresmittel mindestens 50 Masseprozent der im Rahmen der Sammlung der restentleerten Kunststoff-, Metall- und Verbundverpackungen nach $₫ 14$ Absatz 1 insgesamt erfassten Abfälle dem Recycling zuzuführen."

Auch wenn die Nichterfüllung der vorgegebenen Quoten keine Ordnungswidrigkeit nach $\$ 34$ Verpack $G$ darstellt, so geht von diesen Vorgaben durchaus ein beachtlicher Handlungsdruck auf die Dualen Systeme aus. Zudem soll deren Einhaltung gem. $\$ 16$ Abs. 7 VerpackG von der Bundesregierung innerhalb von drei Jahren nach dem 1.1.2022 mit

75) Prelle, in: Schmehl/Klement (Hrsg.), GK-KrWG, \$4 VerpackG, Rdnr. 5.

76) BMU, Bekanntmachung der Erhebungen der Bundesregierung bezüglich des Anteils der in Mehrweggetränkeverpackungen sowie in ökologisch vorteilhaften Einweggetränkeverpackungen abgefüllten Getränke in den Jahren 2004 bis 2014 nach $\ 1$ Abs. 2 S. 2 der Verpackungsverordnung vom 17.8.2016, BAnz. AT 31.8.2016 B4, S. 2.

77) Umweltbundesamt, Verbrauch von Getränken in Mehrweg- und ökologisch vorteilhaften Einweggetränkeverpackungen, UBATexte 106/2019, S. 30.

78) Schlummer, Vortrag „Recycling von Verpackungskunststoffen - Technologien, Herausforderungen und Märkte", Nürnberg 24.9.2019, Stand 21.2.2020, abrufbar unter https//www. ivv.fraunhofer.de/content/dam/ivv/de/documents/info/fachpack-2019-schlummer-vortrag.pdf. 
dem Ziel einer weiteren Erhöhung der materialspezifischen Verwertungsquoten in Abs. 2 S. 1 und 2 und der Recyclingquote in Abs. 4 S. 1 überprüft werden.

$\$ 21$ VerpackG regelt die ökologische Gestaltung der Beteiligungsentgelte. Danach sind die Systeme verpflichtet, besonders recyclingfähige Verpackungen und Verpackungen aus Rezyklaten zu fördern. $\$ 21$ Abs. 2 VerpackG sieht vor, dass die Systeme der Zentralen Stelle (Stiftung Zentrale Stelle Verpackungsregister) und dem Umweltbundesamt jährlich berichten müssen, wie diese Vorgaben umgesetzt wurden, wobei der Recyclinganteil der beteiligten Verpackungen je Materialart eine wesentliche Angabe ist. Die Zentrale Stelle zog am 29.10.2019 nach 10 Monaten Verpackungsregister bereits eine erste positive Bilanz: Die Recyclingziele seien für alle Materialarten übertroffen worden, fast $90 \%$ der Kunststoffverpackungen seien im Inland verwertet worden und nur 0,05\% der Quotenmenge sei in ein Nicht-EU-Land (Schweiz) exportiert und dort nachweislich verwertet worden. ${ }^{79}$

Die Zentrale Stelle hat am 30.8.2019 im Einvernehmen mit dem Bundesumweltamt einen ,Mindeststandard für die Bemessung der Recyclingfähigkeit von systembeteiligungspflichtigen Verpackungen gemäß $\$ 21$ Abs. 3 VerpackG" veröffentlicht. Darin werden unter Ziff. 2 als Mindestkriterien das Vorhandensein einer geeigneten Sortier- und Verwertungsinfrastruktur, die Sortierbarkeit der Verpackung sowie ggf. die Trennbarkeit ihrer Komponenten und das Fehlen von Unverträglichkeiten von Verpackungskomponenten oder enthaltenen Stoffen, die nach der Verwertungspraxis einen Verwertungserfolg verhindern können, benannt. Im Anhang 2 werden für Kunststoffverpackungen Eigenschaften aufgezählt, bei deren Vorhandensein eine Prüfung der Identifizierbarkeit in der sensorgesteuerten Sortierung durch Messung erforderlich ist. Genannt sind dort bekannte Recyclingerschwernisse wie eine großflächige Etikettierung mit Fremdmaterial, Fullsleeve-Etikettierungen, ein Multilayer-Aufbau, die Metallisierung, eine dunkle Farbgestaltung unter Verwendung rußbasierter Farbstoffe sowie unterschiedliche Kunststoffarten auf Vorder- und Rückseiten. Und es wurde ein Regelkreislauf geschaffen, der deutliche Verbesserungen beim Thema Recycling bewirken könnte.

Zusammenfassend ist festzustellen, dass in Deutschland das Recycling von Kunststoffverpackungen einen hohen Stellenwert besitzt und dass Recycling in sehr vielen Vorschriften des KrWG und auch des VerpackG geregelt ist wird. Gleichwohl ist es eine Tatsache, dass trotzdem immer noch nicht oder nur sehr schwer recycelbare Kunststoffverpackungen im Einsatz sind. Es stellt sich die Frage, warum nach wie vor schwarze bzw. dunkle Kunststoffverpackungen (z. B. für Shampoo) in Umlauf gebracht werden dürfen, obwohl diese nach den heute üblichen Sortiertechniken noch nicht recyclingfähig sind; schon die Substitution des rußbasierten Farbstoffs durch „NIR-Black“ (Near Infrared Detectable), wodurch solche Verpackungen von NIRScannern automatisch detektiert werden könnten, würde die Recyclingfähigkeit von 0 auf über $90 \%$ steigern. ${ }^{80}$ Und auch die Problematik der vielen Kunststoffverpackungen aus Mischkunststoffen stellt nach wie vor eine Herausforderung für das Recycling dar.

Der Rechtsrahmen „Recycling“ ist in Deutschland durch das VerpackG und das KrWG, vor allem vor dem Hintergrund der beabsichtigten KrWG Novelle, gut vorbereitet. Es muss beobachtet werden, wie sich deren Anwendung auf das Aufkommen von als kritisch zu beurteilenden Kunststoffverpackungen auswirkt. Auch hier gilt es, durch verbindlichere Regelungen eine gute Recyclingfähigkeit von dafür grundsätzlich geeigneten Kunststoffverpackungen sicherzustellen; und auch in diesem Kontext spielt das Chemikalienrecht eine wichtige Rolle.

\subsection{Rechtsrahmen „Information und Aufklärung“}

Eine besondere Bedeutung im Rahmen einer funktionierenden Kreislaufwirtschaft hat die Mitwirkung der Bevölkerung. Eine möglichst den Vorgaben entsprechende Abfalltrennung und die Vermeidung von Littering sind ebenso wichtig wie bewusste und auf die Vermeidung von unnötigen Abfallmengen zielende Konsumentscheidungen. Um dies zu erreichen, bedarf es der Information und Aufklärung der Verbraucher.

An einer Online-Umfrage von Zeit Online zum Thema richtiges Mülltrennen haben sich rund 470000 Leserinnen und Leser beteiligt. Dabei wurde abgefragt, welche Müllgegenstände in welche Tonne gehören. Die durchschnittliche Fehlwurfquote lag bei $34 \% .{ }^{81} \mathrm{Als}$ Problem wurden z.B. aus mehreren Materialien bestehende Verpackungen genannt: Wer mache vor dem Wegwerfen schon die Pappbanderole von einem Yoghurtbecher ab und entsorge Plastikbecher, Pappbanderole und Aluminiumdeckel getrennt? Laut Branchenexperten dürfte die Fehlwurfquote bei Verpackungsmüll zwischen 40 und $60 \%$ liegen ${ }^{82}$, während die Fehlerquoten bei Papierabfällen nur bei ca. $2 \%$ lägen. Bei der Frage nach den Ursachen scheint die korrekte Zuordnung von Verpackungsabfällen in vielen Fällen nicht ganz einfach zu sein, sodass sehr schnell die Themen Information und Aufklärung im Fokus stehen. Fest steht, dass die Ziele der Kreislaufwirtschaft ohne ausreichende Information und kompetente Beratung nicht $\mathrm{zu}$ realisieren sind.

Diesen Aspekt greift $₫ 46 \mathrm{KrWG}$ auf und verpflichtet die öffentlich-rechtlichen Entsorgungsträger ,zur Information und Beratung über die Möglichkeit der Vermeidung, Verwertung und Beseitigung von Abfällen“. Die Abfallberatungspflicht kann als ein Instrument der informativen Umweltpolitik in einer funktionierenden Kreislaufwirtschaft angesehen werden. ${ }^{83}$ Auf welche Art und Weise Information und Beratung zu erfolgen haben, lässt das Gesetz offen, hier hat der Pflichtige einen Gestaltungsspielraum. Viele Informationen werden heute über das Internet bereitgestellt, aber auch andere Formen wie Informationsveranstaltungen, Informationsmaterial oder Abfallberater sind möglich.

Sehr interessant sind die weitergehenden Ansätze im bereits erwähnten Entwurf eines Gesetzes zur Umsetzung der Abfallrahmenrichtlinie der Europäischen Union. Dieser sieht in $\$ 23$ Abs. 2 Nr. 9 KrWG-E eine erweiterte Produktverantwortung für Entwickler, Hersteller, Be- und Verarbeiter sowie Vertreiber von Erzeugnissen vor. Diese Vorschrift ist auch einschlägig, da es sich bei Kunststoffverpackungen und Einwegkunststoffprodukten um eigenständige Erzeugnisse handelt. Die Produktverantwortung soll danach künftig auch ,die Information und Beratung der Öffentlichkeit über Möglichkeiten der Vermeidung, Verwertung und Beseitigung von Abfällen, insbesondere über Maßnahmen zur Verhinderung der Vermüllung der Umwelt" umfassen. In $\$ 24 \mathrm{Nr}$. 9 KrWG-E wird die Bundesregierung zur Festlegung von Anforderungen nach $\$ 23$ KrWG-E ermächtigt, nach Anhörung der beteiligten Kreise (\$68 KrWG-E) durch Rechtsverordnung mit Zustimmung des Bundesrates zu bestimmen, dass ,für bestimmte Erzeugnisse, insbesondere solche, deren Verwendung in er-

79) Stiftung Zentrale Stelle Verpackungsregister, Positive Bilanz nach 10 Monaten Verpackungsregister - Verpackungsgesetz entfaltet beabsichtigte Wirkungen, Pressemitteilung vom 29. 10. 2019.

80) Christiani, Vortrag „Bewertung der Recyclingfähigkeit von Kunststoffverpackungen“, Nürnberg 24.9.2019, S. 17.

81) Zeit Online, „Wissen Sie, was in welche Tonne gehört?““

82) Süddeutsche Zeitung, Hohe Fehlwurfquote beim Verpackungsmüll.

83) Spiecker gen. Döhmann, in: Schmehl/Klement (Hrsg.), GKKrWG, $\$ 46$ KrWG, Rdnr. 2. 
heblichem Umfang zur Vermüllung der Umwelt beiträgt, die Öffentlichkeit über die Auswirkungen der Vermüllung der Umwelt, die Möglichkeiten der Vermeidung und der Bewirtschaftung der nach Gebrauch der Erzeugnisse entstehenden Abfälle zu beraten und zu informieren" ist. In \25 Abs. 2 Nr. $1 \mathrm{KrWG}-\mathrm{E}$ ist weiter geregelt, dass die Bundesregierung ermächtigt wird, zur Festlegung von Anforderungen nach $\$ 23 \mathrm{KrWG}-\mathrm{E}$ nach Anhörung der beteiligten Kreise ( $\$ 68 \mathrm{KrWG}-\mathrm{E})$ durch Rechtsverordnung mit Zustimmung des Bundesrates zu bestimmen, wer die Kosten für die Beratung und Information nach $\$ 24$ Nummer 9 KrWG-E zu tragen hat.

Auch nach dem Gesetzentwurf können die Abfallwirt-

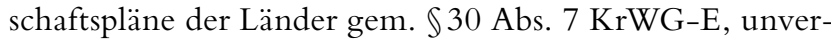
ändert gegenüber dem aktuellen $\$ 30$ Abs. 7 KrWG, den Einsatz von Sensibilisierungskampagnen sowie Informationen für die Öffentlichkeit oder eine bestimmte Verbrauchergruppe enthalten. Als Kann-Vorschrift hat dies allerdings nur eine schwache Verpflichtungswirkung. Auch die Abfallvermeidungsprogramme sollen gem. \$33 Abs. $2 \mathrm{~m}$ KrWG-E u. a. die Entwicklung und Unterstützung von Informationskampagnen, die für Abfallvermeidung und Vermüllung sensibilisieren, vorsehen.

Zusammenfassend ist festzustellen, dass insbesondere die vorgesehenen Neuregelungen im Gesetzentwurf deutlich erweiterte Informations- und Beratungspflichten, insbesondere im Rahmen einer erweiterten Produktverantwortung, enthält, ein deutlicher Fortschritt gegenüber der aktuellen Rechtslage. Die Thematik eignet sich aber auch in besonderer Weise für entsprechende Initiativen aller relevanten Akteure aus dem öffentlich-rechtlichen Bereich (z.B. BMU oder die für Umwelt zuständigen Landesministerien), die auch ohne weitere rechtliche Anpassungen jederzeit möglich sind.

\subsection{Internationale Einbettung und Koordination}

Bislang ist international, trotz der vielen Initiativen auf verschiedenen Ebenen, noch keine vollständig integrierte Vorgehensweise erkennbar. Anders als bei Thema Klima steht es beim Thema Plastikmüll fest, dass der menschliche Einfluss $100 \%$ beträgt. Die Thematik betrifft, ebenso wie das Thema Klima, alle Menschen und das gesamte weltweite Ökosystem und der Handlungsbedarf ist enorm. Daher wäre eine Koordination aller Aktivitäten, idealerweise über die UN, ein wichtiger nächster Schritt. Als Vorbild könnte der gemeinsame Angang beim Thema Klima dienen, wo seit 1988 ein Weltklimarat (IPCC, Intergovernmental Panel on Climate Change) existiert, regelmäBig Weltklimakonferenzen stattfinden und internationale Abkommen geschlossen wurden. Die Bundesrepublik Deutschland ist international über ihre Mitgliedschaften (EU, UN, OECD) oder informelle Beteiligungen (z. B. G7, G20) bestens eingebunden und sollte sich auf allen Ebenen mit einer überzeugenden eigenen Strategie für die notwendigen internationalen Verbesserungen einsetzen.

\section{Ergebnis}

Abschließend soll versucht werden, die eingangs gestellt Frage zu beantworten: Braucht Deutschland ein Kunststoffgesetz? Fest steht, dass ein eigenständiges Gesetz mit allen relevanten Regelungen zur Kunststoffthematik nicht in die bestehende Systematik des europäischen und deutschen Umweltrechts passen würde. Es wären umfangreiche Änderungen auch an vielen anderen Stellen erforderlich. Und es sollte von der praktischen Seite berücksichtigt werden, dass solche grundlegenden Änderungen nur sehr schwer durchsetzbar sind, was die Schicksale des Umweltgesetzbuchs und des Wertstoffgesetzes belegen, die beide nicht eingeführt wurden. Zu berücksichtigen ist aber auch, dass es aktuell kein Kompendium zum Plastikrecht/Kunststoffrecht gibt, das einen Gesamtüberblick bietet. Die gesetzlichen Regelungen sind teilweise sehr kompliziert und nur für Spartenfachleute verständlich. Dies erschwert zielgerichtete gesetzliche Anpassungen erheblich. Die verbindende Klammer könnte eine $\mathrm{zu}$ erarbeitende nationale Kunststoffstrategie bilden. Deutschland besitzt trotz der beschriebenen gesetzlichen Änderungsbedarfe alle Voraussetzungen, um eine solche Strategie auch rechtlich rasch umsetzen zu können. Entscheidend sind der politische Wille und, dass sich die Politik nicht zu sehr von den vielfältigen Lobbyinteressen beeinflussen lässt.

Deutschland braucht somit nicht zwingend ein neues Kunststoffgesetz. Angezeigt ist vielmehr ein ganzheitlicher, politisch-strategischer Ansatz im Zusammenwirken mit der EU, der auch rasch die notwendigen Gesetzesänderungen nach sich ziehen sollte.

Open Access. Dieser Artikel wird unter der Creative Commons Namensnennung 4.0 International Lizenz veröffentlicht, welche die Nutzung, Vervielfältigung, Bearbeitung, Verbreitung und Wiedergabe in jeglichem Medium und Format erlaubt, sofern Sie den/die ursprünglichen Autor(en) und die Quelle ordnungsgemäß nennen, einen Link zur Creative Commons Lizenz beifügen und angeben, $o b$ Änderungen vorgenommen wurden.

Die in diesem Artikel enthaltenen Bilder und sonstiges Drittmaterial unterliegen ebenfalls der genannten Creative Commons Lizenz, sofern sich aus der Abbildungslegende nichts anderes ergibt. Sofern das betreffende Material nicht unter der genannten Creative Commons Lizenz steht und die betreffende Handlung nicht nach gesetzlichen Vorschriften erlaubt ist, ist für die oben aufgeführten Weiterverwendungen des Materials die Einwilligung des jeweiligen Rechteinhabers einzuholen.

Weitere Details zur Lizenz entnehmen Sie bitte der Lizenzinformation auf http://creativecommons.org/licenses/by/4.0/deed.de.

Open Access funding enabled and organized by Projekt DEAL. 